\title{
Análise do conteúdo imagético de nutrição humana em livros didáticos de Ciências aprovados pelo Programa Nacional do Livro Didático 2014
}

\author{
Analysis of human nutrition imagery content in Science textbooks \\ approved by the National Textbook Program 2014
}

André Amaral Gonçalves Bianco`

${ }^{1}$ Curso de Ciências, Universidade Federal de São Paulo (UNIFESP) - Diadema (SP), Brasil.

DOI: http://dx.doi.org/10.7322/abcshs.v40i3.803

\section{RESUMO}

Introdução: As mudanças de hábitos alimentares e estilo de vida da população brasileira nas últimas décadas têm contribuído para o aumento do número de casos de sobrepeso e obesidade. Aeducação nutricional é estratégia fundamental para o enfrentamento desse problema e, no Brasil, é recente a preocupação com ela. Mesmo após décadas de debates sobre esse assunto, ainda é escasso o número de materiais didáticos adequados. Objetivo: Nesta pesquisa objetivou-se analisar quantitativamente e qualitativamente as imagens relacionadas à educação nutricional de livros didáticos aprovados no Programa Nacional do Livro Didático (PNLD). Métodos: Foi realizado um estudo transversal descritivo, no qual se utilizou o método de análise de conteúdo. Foram analisadas nove coleções de livros didáticos de Ciências aprovadas pelo PNLD 2014. Resultados: A análise de nove coleções de livros didáticos de Ciências aprovados pelo PNLD 2014 revelou falhas no conteúdo imagético. Foram encontradas 328 imagens com distinção para os temas anatomia e fisiologia do sistema digestório, em detrimento da apresentação de conteúdos relacionados ao sobrepeso e à obesidade. Em relação às patologias associadas à nutrição, ainda são enfatizadas patologias tradicionalmente apresentadas nos livros didáticos, como o bócio. Conclusão: Há necessidade de revisão do conteúdo imagético presente nos livros didáticos para que este sirva de contribuição ao combate do sobrepeso e da obesidade.

Palavras-chave: materiais de ensino; ensino fundamental e médio; nutrição da criança.

\section{ABSTRACT}

Introduction: Changes in eating habits and lifestyle of the population in recent decades have contributed to the increased number of cases of overweight and obesity. Nutrition education is strategically important to face this problem and in Brazil is recent concern for nutrition education. Even after decades of debate on this subject is still scarce the number of suitable teaching materials. Objective: In this study we aimed to analyze quantitatively and qualitatively the images of nutrition education textbooks approved in the National Textbook Program (PNLD). Methods: A descriptive cross-sectional study in which we used the method of content analysis. Nine collections of Sciences textbooks approved by PNLD 2014 were analyzed. Results: The analysis of nine collections of Sciences textbooks approved by the PNLD 2014 revealed flaws in imagery content. 328 images with distinction were found to the issues anatomy and physiology of the digestive system, rather than submitting to the related overweight and obesity content. Regarding the diseases associated with nutrition, they are still emphasized pathologies traditionally presented in textbooks, such as goiter. Conclusion: There is a need for revision of this imagery content in textbooks to have it as a contribution to the fight against overweight and obesity.

Keywords: teaching materials; education, primary and secondary; child nutrition. 


\section{INTRODUÇÃO}

A educação nutricional constitui estratégia fundamental para o enfrentamento dos problemas alimentares e nutricionais encontrados na atualidade ${ }^{1}$. Esses problemas tendem a se agravar pela tendência que vem sendo observada nos padrões de consumo de alimentos ${ }^{2}$.

Entretanto, no Brasil, a educação nutricional foi pouco valorizada como disciplina e como estratégia de política pública até a década de 1990, quando pesquisas ${ }^{3,4}$ mostraram que o aumento das doenças crônicas degenerativas estavam relacionadas aos hábitos alimentares da população $0^{5}$.

Embora a relevância da educação nutricional seja reconhecida, ela se dilui no conjunto de propostas pelo fato de suas bases teórico-conceituais e operacionais não estarem claramente estabelecidas ${ }^{6}$.

Triches e Giugliani ${ }^{7}$ concluíram que a obesidade está associada a menos conhecimentos de nutrição e práticas alimentares, e crianças com essas características apresentam cinco vezes mais chances de serem obesas. Entretanto, não se deve conceber a prática da educação nutricional apenas na transmissão de conceitos de nutrição, pois estudos não encontraram diferenças significativas entre conhecimentos de conceitos de nutrição de crianças e adolescentes obesos e eutróficos ${ }^{8,9}$.

Apesar de ter sido detectada a progressão da transição nutricional no Brasil, caracterizada pela redução na prevalência dos déficits nutricionais, para ocorrência do sobrepeso e obesidade ${ }^{10}$, a carência nutricional coexiste com o sobrepeso e a obesidade ${ }^{11}$.

A partir de 1974/1975 inicia-se a elevação epidêmica do sobrepeso e da obesidade em adultos. A obesidade em homens adultos praticamente triplicou entre a metade dos anos 1970 e o início dos anos 2000 , aumentando em mais de $50 \%$ nas mulheres ${ }^{11}$. Portanto, aspectos regionais e desigualdades sociais que comprometem o acesso da população a uma alimentação adequada devem ser considerados $^{12}$, mas não invalidam a investigação em educação nutricional concernente ao combate do sobrepeso e da obesidade.

Segundo o Ministério da Saúde ${ }^{13}$ e Batista Filho ${ }^{11}$, a escola deve disseminar informações de saúde durante o Ensino Básico. Entretanto, pesquisadores brasileiros ${ }^{14,15}$ observaram conceitos sobre nutrição desatualizados e incompletos em textos de livros didáticos de Ciências.

Os livros didáticos são os recursos pedagógicos mais usuais e valorizados por professores e estudantes e, para muitos brasileiros, eles representam o único texto com que interagem ao longo da vida ${ }^{16}$. Uma vez que as imagens presentes nos livros auxiliam a sua função pedagógica, é pertinente analisar o conteúdo conceitual contido nessas imagens.

As imagens são representações da realidade e pode-se pensar nelas como referências ao mundo ou objeto que ela pretende descrever ${ }^{17}$. Sua importância no Ensino de Ciências é tão grande que Amador ${ }^{18}$ considerou a evolução da Geologia dependente do desenvolvimento de formas de representações visuais mais adequadas.
Apesar das análises criteriosas realizadas pelo Ministério da Educação (MEC) e da melhora progressiva que vem ocorrendo nos livros didáticos no Brasil, Schall ${ }^{19}$ alega que os livros didáticos aprovados pelo Programa Nacional do Livro Didático (PNLD) ainda apresentam conteúdos e ilustrações inadequadas e insuficientes.

Nesse contexto, foi investigado o conteúdo imagético de livros didáticos de Ciências do Ensino Fundamental II, aprovados pelo PNLD 2014, com o objetivo de analisar a frequência e a qualidade do conteúdo das imagens relacionadas à Nutrição Humana.

\section{MÉTODOS}

Foi realizado um estudo transversal descritivo, no qual se utilizou o método de análise de conteúdo ${ }^{20}$. Foram analisadas nove coleções de livros didáticos de Ciências aprovadas pelo PNLD 2014. O instrumento de análise da qualidade das informações apresentadas pelas imagens relacionadas à Nutrição foi elaborado após revisão da literatura, realizada entre fevereiro e julho de 2015, referente aos hábitos alimentares de consumo, ao estado nutricional e à saúde de estudantes do Ensino Fundamental II brasileiro, à transição nutricional, à alimentação saudável, à promoção de saúde e ao livro didático. Os idiomas utilizados para a pesquisa foram português, inglês e espanhol. As bases de dados pesquisadas foram: Medline, da National Library of Medicine, Scientific Eletronic Library Online (SciELO) e Literatura Latino Americana e do Caribe em Ciências da Saúde (LILACS).

A revisão bibliográfica possibilitou a escolha de sete unidades de análise, consideradas impreteríveis ao desenvolvimento e manutenção de hábitos alimentares saudáveis:

1. consumir frutas e vegetais diariamente;

2. evitar o consumo excessivo de doces e gorduras ${ }^{1}$;

3. beber quatro ou mais copos de água por dia;

4. evitar o consumo excessivo de sal de cozinha ${ }^{2}$;

5. realizar de cinco a seis refeições por dia, contando os lanches;

6. comer devagar, mastigando bem os alimentos;

7. substituir o consumo de refrigerantes e outras bebidas adocicadas pelo consumo de sucos naturais.

Os dados coletados foram analisados com base nas abordagens quantitativa - a ocorrência dessas informações foi classificada como "ausente" ou "presente" - e qualitativa - em concordância com os conhecimentos atuais em Nutrição Humana. A partir da ficha de avaliação, os dados brutos foram organizados em tabelas de contingência, com utilização de estatística descritiva, com distribuição simples e cálculo de frequência relativa.

A American Dietetic Association ${ }^{21}$ considera adequado para crianças de 2 a 11 anos o consumo de doces inferior a $25 \%$ da ingesta calórica diária total e, para a faixa etária dos 4 aos 18 anos, o consumo de gorduras entre 25 e 35\% da ingesta calórica diária total. Para indivíduos na faixa etária dos 9 aos 18 anos, a ingestão diária limite de sódio deve ser de $1,5 \mathrm{~g}^{22}$. 
Foram encontrados diversos trabalhos na literatura especializa$\mathrm{da}^{23-27}$ que preconizam a adoção dessas sete orientações por estudantes e suas famílias, sendo os indivíduos obesos ou não.

De modo geral, consideraram-se adequadas imagens que levassem o estudante a pensar, que orientassem o deslocamento do consumo de alimentos pouco saudáveis (como enlatados, congelados, pré-cozidos e fast food) para alimentos considerados saudáveis (frutas, cereais, leguminosas, entre outros) e que auxiliassem a ampliar a capacidade de escolha dos estudantes acerca de suas refeições.

A despeito de reconhecer a importância da prática de atividades físicas para uma vida saudável, com redução do risco de desenvolvimento de Doenças Crônicas Não Transmissíveis (DCNT), o foco desta pesquisa foi a análise exclusiva do conteúdo de Educação Nutricional presente nos livros didáticos analisados.

\section{RESULTADOS}

As nove coleções analisadas apresentaram tópicos referentes ao tema Nutrição Humana. Esses tópicos estavam presentes em livros do oitavo ano - oito das nove coleções analisadas - e do sexto ano - em apenas uma das coleções investigadas.
Foi encontrado um total de 328 figuras relacionadas à Nutrição Humana, distribuídas equitativamente entre os nove livros. Dois desses livros apresentaram diferenças quantitativas significativas - o maior número de figuras encontrado em um único livro foi 46 e o menor 22; os demais apresentaram uma média de 37 figuras por livro. Nesta análise não foram contabilizados gráficos e tabelas.

A distribuição quantitativa das figuras por temas mais frequentes pode ser observada na Tabela 1 .

Na Tabela 2 encontram-se as porcentagens de ocorrências das figuras, divididas por tema, em relação ao número de figuras do respectivo livro e das figuras dos livros em relação ao total de figuras encontradas nas nove coleções analisadas.

A somatória da coluna "geral", da Tabela 2, perfaz $84 \%$, pois foram encontradas figuras relativas a temas pontuais que representaram $16 \%$ do total de figuras encontradas nos livros analisados. Esses temas foram: ingestão e gasto calórico (1\%), ação enzimática (2\%), procedimentos experimentais (1\%), aditivos alimentares (1\%), alimentos transgênicos $(0,3 \%)$, aleitamento materno $(0,6 \%)$, desperdícios de alimentos $(0,6 \%)$, açúcar refinado $(0,3 \%)$, alimentos diet e light $(0,6 \%)$, doença celíaca $(0,3 \%)$, intolerância à lactose $(0,3 \%)$, tiras cômicas $(1 \%)$,

Tabela 1: Distribuição quantitativa das figuras relacionadas aos temas de Nutrição Humana mais frequentemente encontrados nos livros didáticos analisados

\begin{tabular}{|c|c|c|c|c|c|c|c|c|}
\hline \multicolumn{9}{|c|}{ Temas } \\
\hline Coleções & $\begin{array}{l}\text { Grupos de } \\
\text { alimentos }\end{array}$ & $\begin{array}{l}\text { Sistema } \\
\text { digestório }\end{array}$ & $\begin{array}{l}\text { Conservação } \\
\text { dos alimentos }\end{array}$ & $\begin{array}{c}\text { Patologias associadas } \\
\text { à Nutrição }\end{array}$ & $\begin{array}{l}\text { Saúde } \\
\text { bucal }\end{array}$ & $\begin{array}{l}\text { Pirâmide } \\
\text { alimentar }\end{array}$ & $\begin{array}{l}\text { Informações } \\
\text { nutricionais }\end{array}$ & Total \\
\hline 1 & 2 & 10 & - & - & 1 & - & - & 13 \\
\hline 2 & 9 & 13 & 4 & 2 & 5 & - & 3 & 36 \\
\hline 3 & 6 & - & 15 & 3 & - & 1 & - & 25 \\
\hline 4 & 2 & 14 & - & 8 & - & 1 & - & 25 \\
\hline 5 & 19 & 10 & - & 4 & 3 & - & 1 & 37 \\
\hline 6 & 4 & 12 & 3 & 3 & 2 & 1 & - & 25 \\
\hline 7 & 13 & 13 & - & 5 & 2 & - & - & 33 \\
\hline 8 & 9 & 13 & 3 & 3 & 4 & 1 & 6 & 39 \\
\hline 9 & 14 & 14 & 5 & 3 & 2 & 2 & 1 & 41 \\
\hline Total & 78 & 99 & 30 & 31 & 19 & 6 & 11 & 274 \\
\hline
\end{tabular}

-: ausência de imagens no livro para o respectivo tema.

Tabela 2: Porcentagem de ocorrência de imagens relacionadas à Nutrição Humana, em relação à obra e ao total de livros analisados (geral)

\begin{tabular}{|c|c|c|c|c|c|c|c|c|}
\hline \multicolumn{9}{|c|}{ Temas } \\
\hline Coleções & $\begin{array}{l}\text { Grupos de } \\
\text { alimentos }\end{array}$ & $\begin{array}{c}\text { Sistema } \\
\text { digestório }\end{array}$ & $\begin{array}{l}\text { Conservação } \\
\text { dos alimentos }\end{array}$ & $\begin{array}{c}\text { Patologias associadas } \\
\text { à Nutrição }\end{array}$ & $\begin{array}{l}\text { Saúde } \\
\text { bucal }\end{array}$ & $\begin{array}{l}\text { Pirâmide } \\
\text { alimentar }\end{array}$ & $\begin{array}{l}\text { Informações } \\
\text { nutricionais }\end{array}$ & Geral \\
\hline 1 & 15 & 77 & - & - & 8 & - & - & 4 \\
\hline 2 & 25 & 36 & 11 & 6 & 14 & - & 8 & 11 \\
\hline 3 & 24 & - & 60 & 12 & - & 4 & - & 8 \\
\hline 4 & 8 & 56 & - & 32 & - & 4 & - & 8 \\
\hline 5 & 51 & 27 & - & 11 & 8 & - & 3 & 11 \\
\hline 6 & 16 & 48 & 12 & 12 & 8 & 4 & - & 8 \\
\hline 7 & 39 & 3 & - & 15 & 6 & - & - & 10 \\
\hline 8 & 23 & 33 & 8 & 8 & 10 & 3 & 15 & 12 \\
\hline 9 & 34 & 34 & 12 & 7 & 5 & 5 & 2 & 12 \\
\hline
\end{tabular}

-: ausência de imagens no livro para o respectivo tema. 
representações de obras de arte (2\%) e imagens meramente ilustrativas (5\%).

Foram consideradas imagens meramente ilustrativas aquelas cujo conteúdo não fazia relação ao texto ou que fosse dispensável à sua compreensão. Nessa categoria, encontraram-se imagens como a fotografia de uma criança comendo um pedaço de melancia; de um sorriso; de uma família se alimentando; de uma mulher bebendo água, entre outras.

Com relação as sete unidades de análise propostas neste trabalho, não foram encontradas imagens relacionadas a quatro delas: consumo excessivo de sal (unidade 4), realização de cinco a seis refeições por dia (unidade 5), mastigação lenta dos alimentos (unidade 6) e substituição dos refrigerantes e de outras bebidas adocicadas por sucos naturais (unidade 7).

Imagens relativas a tópicos considerados relevantes à Nutrição e destacados tanto em trabalhos científicos quanto nos meios de comunicação, como gorduras trans, alimentos orgânicos e diabetes, não foram encontradas nos livros analisados. Em contrapartida, imagens relacionadas ao bócio (cuja incidência na população brasileira é relativamente baixa) representaram 13\% das imagens de patologias associadas à Nutrição.

\section{DISCUSSÃO}

Nem toda imagem e nem toda relação texto-imagem é igualmente eficiente em promover a aprendizagem ${ }^{28}$. Portanto, a análise quantitativa de imagens em uma obra didática não é suficiente para sustentar conclusões a respeito de sua qualidade. Nesta pesquisa, a análise quantitativa serviu para investigar a elegibilidade dos autores de livros didáticos das imagens em seus textos. As escolhas dos autores podem estar relacionadas à compreensão da importância de determinados conteúdos ou à ausência de critérios para a escolha das imagens. No primeiro caso, exigências para a aprovação na avaliação do PNLD, como a presença de História da Ciência nos livros didáticos, podem ter contribuído para a escolha de imagens pelo autor. Neste trabalho, foram encontradas representações de obras de arte, de cunho histórico, cuja relevância para a educação nutricional é questionável, mas que podem ser interpretadas como responsivas à necessidade de conteúdos de História da Ciência.

As imagens encontradas nas nove coleções analisadas guardam grande semelhança, o que restringe as opções do professor por materiais distintos. Da mesma forma, a preferência por restringir o conteúdo, e consequentemente as imagens, de nutrição humana ao oitavo ano não dá margem para o professor trabalhar o assunto em outros anos do Ensino Fundamental II.

A análise do conteúdo científico das imagens revelou pouca adequação às unidades de análise adotadas nesta pesquisa. Embora os autores sejam conscientes da importância da educação nutricional, pois esse conteúdo foi encontrado em todas as coleções analisadas, eles deram pouca ênfase imagética ao sobrepeso, à obesidade e ao consumo excessivo de sal. Isso pode representar um déficit no aprendizado dos alunos, pois compromete a sua reflexão acerca de temas nutricionais contemporâneos.

Mesmo para as imagens que contemplaram as unidades de análise (1), (2) e (3), o conteúdo não deixava claras quais são as recomendações diárias para o consumo de frutas e verduras, o que pode ser considerado consumo excessivo de doces e gorduras e quais as possíveis consequências do não atendimento a essas recomendações. Com relação à unidade (3), em nenhuma das imagens encontradas estavam disponíveis as recomendações de ingesta diária de água. Elas trataram exclusivamente de apresentar imagens de indivíduos bebendo água.

O número de imagens meramente ilustrativas pode ser considerado alto, sobretudo porque o emprego desse tipo de imagem não chega a ter valor didático e o uso de ilustrações que embelezem o texto não melhora a aprendizagem da informação ${ }^{29}$. Esse foi um recurso adotado nos livros didáticos de português a partir da década de 1960 - a imagem empregada dessa forma é redundante em relação ao texto, não acrescenta ou renova diferentes leituras e não tem a preocupação em dialogar com o texto ${ }^{30}$, pois, naquele momento, acreditava-se que essas imagens motivariam o aluno à leitura.

De modo geral, os autores adotaram imagens que reforçam ou confirmam as ideias presentes no texto, fazendo uso simples e óbvio delas, não as utilizando como estímulo à reflexão.

É necessário que o professor faça uma análise dos livros didáticos com os quais irá trabalhar, mesmo se tratando de livros aprovados pelo PNLD, que passaram por avaliação de comissão técnica, pois o livro didático é o principal mediador de conceitos científicos do qual o professor se apropria e desempenhará a função de conduzir o aprendizado dos alunos.

Os resultados da presente pesquisa evidenciam a necessidade de aprimoramento imagético dos livros didáticos analisados, com adequação dos conteúdos e da frequência de ocorrência. A escolha das imagens dos livros didáticos deve desprezar aspectos ilustrativos e priorizar a transmissão de informações relevantes e relacionadas ao texto.

A imagem no livro didático não é apenas uma alegoria. Ela apresenta valor cognitivo e auxilia na construção de modelos mentais, portanto, os autores de livros didáticos devem buscar empregá-las de modo que permita a expressão e o desenvolvimento dessas potencialidades.

O professor deve procurar materiais didáticos complementares para a elaboração imagética de suas aulas, sobretudo quando estes utilizam o expediente de apresentar imagens presentes em livros didáticos em suas apresentações multimídia em sala de aula. Ele deve selecionar imagens de diferentes fontes, priorizando aquelas com potencial educativo, em detrimento de imagens meramente ilustrativas, visando, com isso, aperfeiçoar o desenvolvimento da prática pedagógica restrita ao uso do livro didático. 


\section{REFERÊNCIAS}

1. World Health Organization (WHO). Physical Status: the use and interpretation of anthropometry. Report of a WHO expert commitee. Technical Report Series, n.854. Geneva: WHO; 1995.

2. Boog MCF. Educação nutricional em serviços públicos de saúde. Cad Saúde Pública. 1999;15(supl.2):S139-47. http://dx.doi.org/10.1590/S0102-311X1999000600014

3. Coitinho DC, Leão MM, Recine E, Sichieri R. Condições nutricionais da população brasileira: adultos e idosos. Brasília: INAN; 1991. p. 39.

4. Instituto Brasileiro de Geografia e Estatística (IBGE) [Internet]. Pesquisa de orçamento familiar. Disponível em: http://www.ibge. gov.br/home/estatistica/populacao/condicaodevida/pof/default. shtm. Acesso em: 23 jun. 2015.

5. Boog MCF [Internet]. Educação nutricional: Por que e para que? J Unicamp. 2004. Disponível em: http://www.unicamp.br/unicamp/ unicamp_hoje/jornalPDF/ju260pag02.pdf. Acesso em: 23 jun. 2015.

6. Santos LAS. Educação alimentar e nutricional no contexto da promoção de práticas alimentares saudáveis. Rev Nutr. 2005;18(5):681-92.

http://dx.doi.org/10.1590/S1415-52732005000500011

7. Triches RM, Giugliani ERJ. Obesidade, práticas alimentares e conhecimentos de nutrição em escolares. Rev Saúde Pública. 2005;39(4):541-47.

http://dx.doi.org/10.1590/S0034-89102005000400004

8. Thakur N, D'Amico F. Relationship of nutrition knowledge and obesity in adolescence. Fam Med. 1999;31(2):122-7.

9. Reinehr T, Kersting DM, Chahda C, Wollenhauot A, Andler W. Nutritional knowledge of obese and non obese children. J Pediatr Gastroenterol Nutr. 2001;33(3):251.

10. Wang Y, Monteiro CA, Popkin BM. Trend of obesity and underweight in older children and adolescentes in the USA, Brazil, China and Russia. Am J Clin Nutr. 2002;75(6):971-7.

11. Batista Filho M, Souza AL, Miglioli TC, Santos MC. Anemia e obesidade: um paradoxo da transição nutricional brasileira. Cad Saúde Pública. 2008;24(Suppl2):S247-57. http://dx.doi.org/10.1590/S0102-311X2008001400010

12. Rodrigues LPF, Roncada MJ. Educação nutricional no Brasil: evolução e descrição de proposta metodológica para escolas. Com Ciências Saúde. 2008;19(4):315-22.

13. Brasil. Ministério da Saúde. Secretaria de atenção à saúde. Departamento de atenção básica. Guia alimentar para a população brasileira; promovendo a alimentação saudável. Brasília: Ministério da Saúde; 2008.

14. Gaglianone CP. Estudo do conteúdo relacionado à nutrição em livros didáticos de Ciências utilizados no ensino fundamental brasileiro. Dissertação (Mestrado) - Universidade Federal de São Paulo, São Paulo, 1999.

15. Linden SLR. Educação alimentar e nutricional no ensino fundamental: conexões ou desconexões? Dissertação (Mestrado) - Universidade do Vale dos Sinos, Novo Hamburgo, 1999.
16. França VH, Margonari C, Schall VT. Análise do conteúdo das leishmanioses em livros de Ciências e Biologia indicados pelo Programa Nacional de Livros Didáticos (2008/2009). Ciênc Educ. 2011;17(3):625-44.

http://dx.doi.org/10.1590/S1516-73132011000300007

17. Ducrot O. Referente. EnciclopédiaEinaudi. Linguagem-Enunciação. v.2. Lisboa: Imprensa Nacional Casa da Moeda; 1984.

18. Amador F. As imagens no ensino da Geologia. Formação de professores: cadernos didácticos. Série ciências. v. 2. Aveiro: Universidade de Aveiro, 1998.

19. Schall VT. Educação e divulgação científica sobre moluscos de importância médica: breve análise de materiais informativos sobre esquistossomose. In: Encontro Brasileiro de Malacologia. Rio de Janeiro: 2010. p. 391-403

20. Campos CJG. Método de análise de conteúdo: ferramenta para a análise de dados qualitativos no campo da saúde. Rev Bras Enferm. 2004;57(5):611-14.

http://dx.doi.org/10.1590/S0034-71672004000500019

21. Nicklas T, Johnson R, American Dietetic Association. Position of the American Dietetic Association: dietary guidance for healthy children ages 2 to 11 years. J Am Diet Assoc. 2004; 104(4):660-77.

http://dx.doi.org/10.1016/j.jada.2004.01.030

22. Institute of Medicine. Panel on Dietary Reference Intakes for Electrolytes and Water. Dietary Reference Intakes for Water, Potassium, Sodium, Chloride, and Sulfate. Washington: National Academy of Sciences; 2004.

23. Birch LL. Childhood Overweight: Family environmental factors. In: Chen C, Dietz WH, editors. Obesity in Childhood and adolescence. Philadelphia: Lippincot Willians \& Wilkins; 2002. p. 207-22.

24. Dietz WH. Childhood obesity. In: Shils ME. Modern Nutrition in Health and Disease. Baltimore: Williams \& Willians; 1999. p. 1071-80.

25. Dietz WH. Policy and environmental changes related to the prevention and treatment of childhood and adolescent obesity. In: Chen C, Dietz WH. Obesity in Childhood and Adolescence. Philadelphia: Lippincot Williams and Wilkins, 2002. p. 273-86.

26. Bellizzi MC, Dietz WH. Workshop on childhood obesity: summary of the discussion. Am J Clin Nutr. 1999;70(1 part 2):S173-5.

27. Williams CL, Gulli MT, Deckelbaum RJ. Prevention and treatment of childhood obesity. Curr Atheroscler Rep. 2001;3(6):486-97.

28. Mayer RE. Introduction to multimedia learning. 2005a. In: Mayer RE. The Cambridge handbook of multimedia learning. Cambridge: Cambridge Universty Press; 2005. p. 31-48.

29. Levie W, Lentz R. Effects of text illustrations: a review of Research. Res Educ Communic Technol J. 1982;30(4):195-232. http://dx.doi.org/10.1007/BF02765184

30. Belmiro CA. A imagem e suas formas de visualidade nos livros didáticos de Português. Educ Soc. 2000;21(72):11-31. 